\title{
Transmission Interference Improvement of Railway Communication via Distributed Antennas System
}

\author{
Siyu Lin*, Zhangdui Zhong*, Bo Ai*, and Cesar Briso-Rodríguez ${ }^{\dagger}$ \\ ${ }^{*}$ State Key Laboratory of Rail Traffic Control and Safety \\ Beijing Jiaotong University, 100044, Beijing, China \\ †Escuela Universitaria de Ingeniería Técnica de Telecomunicación \\ Universidad Politécnica de Madrid, 28031, Madrid, Spain \\ E-mail: kevinlin1225@gmail.com
}

\begin{abstract}
Transmission interference is a key performance indicator of railway digital mobile communication system (RDMCS), whose performance will become worse in high-speed railway. The factors that influence the transmission interference of RDMCS are analyzed. Based on the relationship between speed and transmission interference, the scheme based on distributed antennas system (DAS) is proposed to improve transmission interference of RDMCS. The proposed scheme can reduce the collision probability between data transmission and handover with the improved performance of transmission interference. Effectiveness of the proposed scheme is verified by simulations and theoretical analysis.

Index Terms - high-speed railway; railway digital mobile communication system(RDMCS); transmission interference; distributed antennas system(DAS)
\end{abstract}

\section{INTRODUCTION}

Presently, High-speed Railway is developing rapidly. Railway digital mobile communication system (RDMCS) is the important part which bears various services, such as train control message transmission and dispatching voice communication. Transmission interference is an important quality of services (QoS) indicator of train control message transmission services which is defined as the number of transmission interference occurrences within a period of time. It is used to describe the impact of RDMCS frame error rate (FER) performance on train control message transmission. Transmission interference indicators consist of two parameters: transmission interference period $\left(\mathrm{T}_{\mathrm{TI}}\right)$ and transmission error-free period (transmission recovery period $\mathrm{T}_{\mathrm{REC}}$ ). $\mathrm{A} \mathrm{T}_{\mathrm{TI}}$ denotes the time period that 30 bytes train control message can not be correctly transmitted under normal communication circumstance. Transmission interference occurs when there is frame error or package loss in train control message received by onboard equipment. $\mathrm{T}_{\mathrm{TI}}$ must be followed by a period within which train control message is correctly transmitted to onboard equipment, which is called $\mathrm{T}_{\mathrm{REC}}$.

The transmission interference performance is hard to meet the requirement in normal-speed railway [1]. However, with the increasing speed of train, doppler shift of radio signals

This work is supported by the Joint State Key Program of the National Natural Science Foundation of China (No. 60830001) and supported by the State Key Laboratory of Rail Traffic Control and Safety (No. RCS2008ZZ006), Beijing Jiaotong University. will increase, and handover will become more frequently. These changes will cause negative impacts on transmission interference performance of RDMCS. Present studies of train control message transmission services still focus on the test methods and the application purpose [2], [3]. QoS indicator requirements are developed based on engineering experience with theoretical analysis [4]. When the train speed increases a great deal, it is imperative to solve how to fulfill the highly efficient and reliable transmission requirements of train control message.

Firstly, the principal elements to have great influence on the transmission interference of RDMCS is presented. Based on the presented elements, this paper proposes the scheme of radio coverage along the railway by distributed antennas system (DAS) with the purpose of improving transmission interference performance under the high-speed condition. We investigate the feasibility of reducing transmission interference by the scheme of distributed antennas system coverage. The performance indicators and carrier interference ratio are analyzed as well.

\section{Principal Elements That AfFect Transmission INTERFERENCE}

RDMCS transmission interference performance is closely related to safety and reliability of train control system. It is important to grasp the principal of influential factor of transmission interference to improve system performance and to ensure the safe operation of high-speed trains.

\section{A. Transmission Interference Requirements of RDMCS}

Transmission interference indicators are formulated based on train control system and characteristics of RDMCS. Before the principal elements affecting transmission interference are analyzed, the requirements of RDMCS transmission interference will be introduced.

Reference [5] defines the worst case of transmission interference, in which handover is accompanied by pre-emption service at the same time. Under this circumstance, a lower priority call has to be released in the selected radio cell before handover can be completed. Data transmission in the handing over radio cell will proceed. During this process, QoS of the present cell will be getting worse and worse, while QoS of 
the switched cell will be getting better and better. The whole process takes about $900 \mathrm{~ms}$, namely release of the pre-empted call delay $(600 \mathrm{~ms})$ and handover time $(300 \mathrm{~ms})$. However, call release delay and handover time are both statistical mean value, therefore, $\mathrm{T}_{\mathrm{TI}}$ should be $<0.8 \mathrm{~s}(95 \%)$ and $<1$ s $(99 \%)$ to satisfy a statistical characteristics.

$\mathrm{T}_{\mathrm{REC}}$ is used to retransmit the wrong and the waiting data clustering after $\mathrm{T}_{\mathrm{TI}}$. The minimum $\mathrm{T}_{\mathrm{REC}}$ must ensure at least the transmission of a list of complete train control message. Normally, the length of train control message is from 250 bytes to 500 bytes. 500 bytes transmission takes $7 \mathrm{~s} \mathrm{~T}_{\mathrm{REC}}$. The largest probable $\mathrm{T}_{\mathrm{REC}}$ is the interval between two normal handovers. Assume that train speed is $360 \mathrm{~km} / \mathrm{h}$, and the diameter of cell is normally larger than $2 \mathrm{~km}$, and then the time interval of handover is about 20s. Therefore, $\mathrm{T}_{\text {REC }}$ should be $<20$ s $(95 \%)$ and $<7$ s $(99 \%)$.

\section{B. Impact of Train Control System Characteristic on Trans- mission Interference}

Transmission interference is caused by a variety of reasons. Impact of train control system characteristic on transmission interference is mainly embodied in handover probability during train control message transmission process, as is shown in Fig. 1.

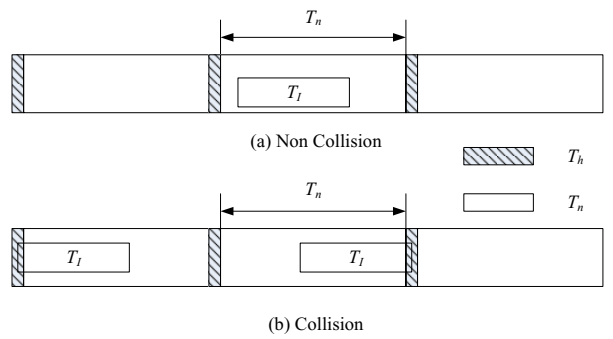

Fig. 1. Collision between data transmission and handover

Fig. 1 (a) indicates that message transmission is completed without collision within the time interval of handover. Fig. 1 (b) indicates two kinds of collision: train control system requires message transmission during handover; handover is required when message transmission have not been completed. Shade area in Fig. 1 denotes handover necessary time $T_{h}$, and handover time interval is $T_{n}$. Assume that the time of train crossing one cell is $T_{c}$. Then,

$$
\begin{gathered}
T_{c}=D / V \\
T_{c}=T_{h}+T_{n}
\end{gathered}
$$

where $D$ denotes cell diameter, $V$ denotes train speed. Assume that train control message transmission time is $T_{I}$, train control message transmission period equal the time of train crossing one cell $T_{c}$, and train control transmission starting time follows uniform distribution. Then, the collision probability within this cell is

$$
p=\frac{T_{I}+T_{h}}{T_{c}}
$$

Collision probability is impacted by train control message transmission characteristics, including length of train control message, message transmission protocol, and message transmission period.

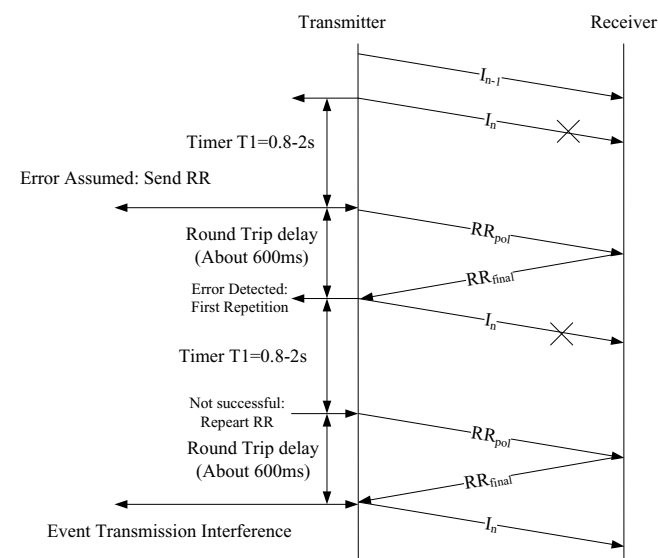

Fig. 2. Data retransmission mechanism

Link layer protocol of high-speed railway train control system employs a protocol similar to high-level data link control (HDLC) protocol. Data frame retransmission mechanism is shown in Fig. 2. The $n$th data frame $I_{n}$ is transmitted when the $n-1$ th data frame $I_{n-1}$ was correctly received, but transmitter has not received the acknowledgement (ACK) message of the correct reception of data frame $I_{n}$, and then RR frame will be transmitted to confirm whether the channel state is good or not after the timer T1 overflow. Data frame $I_{n}$ will be retransmitted after receiving the ACK of RR frame correctly. The reason of transmission interference occurence is that $\mathrm{T}_{\mathrm{TI}}$ is too long or $\mathrm{T}_{\mathrm{REC}}$ is too short. Data frame retransmission will prolong the train control message transmission delay and increase the load of channel. When protocol similar to HDLC is employed to transmit data frame, the message is divided into message information frame of 30 bytes to transmit. This protocol has retransmission mechanism to ensure the accurate arrival of the frame. The complete transmission time of the message is

$$
T_{I}=\frac{L_{I}}{R_{I}}+\frac{L_{I}}{30}(1+F E R) T_{d}+\frac{L_{I}}{30} \times F E R \times T_{r}
$$

where $L_{I}$ and $R_{I}$ denotes the length of train control message and the message transmission rate, respectively. $T_{d}$ denotes the end-to-end transmission delay caused by the transmission of each 30 bytes data frame. $F E R$ is frame error rate, and $T_{r}$ is the waiting delay (including the ACK waiting time of transmission terminal and the transmission time of RR test frame) caused by frame error and frame loss.

If Point-to-Point Protocol (PPP) is selected to transmit data frame. It needs no retransmission mechanism. Assume that end-to-end transmission delay is proportional to message length, and then the complete message transmission time is

$$
T_{I}=\frac{L_{I}}{R_{I}}+\frac{L_{I}}{30} T_{d}
$$

Therefore, transmission interference of RDMCS is impacted by length of train control message, message transmission protocol, and message transmission period. 


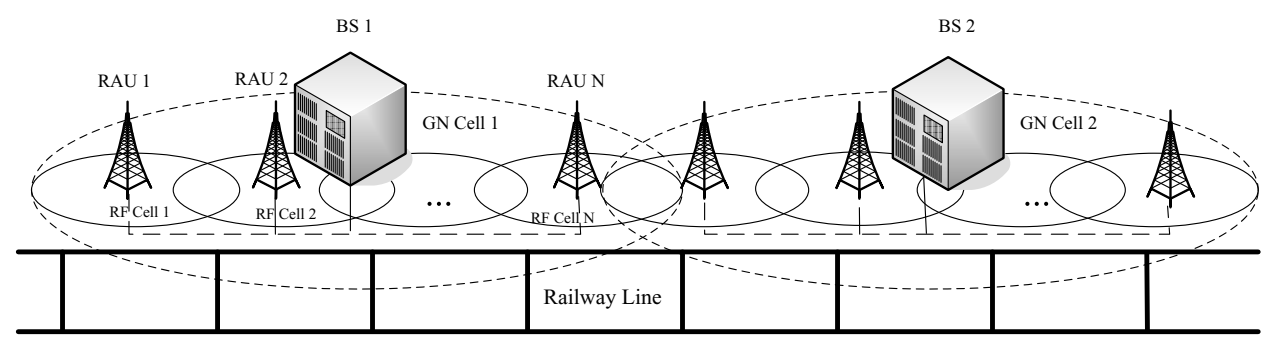

Fig. 3. Distributed antennas coverage and base station single antenna coverage

C. Impact of RDMCS Characteristic on Transmission Interference

RDMCS characteristic affects the transmission interference of the system itself mainly in two aspects, namely wireless channel random fading feature and handover frequency. With the increasing of train speed and the reduction of wireless channel coherent time, the time-varying characteristic will become apparent. Transmission interference caused by wireless channel time-varying characteristic can be efficiently improved by employing equalization algorithm and channel coding algorithm.

Presently, RDMCS handover is a hard handover technique, and there is short-term interruption of data transmission during handover process. Therefore, handover is also an important reason for transmission interference. Handover frequency is determined by train speed and cell coverage area. With the increasing of train speed, handover frequency will grow, which will increase the collision probability and the transmission interference performance will become worse. The relation between collision probability and train speed is expressed in Eq. 6.

$$
p=\frac{\left(T_{I}+T_{h}\right) V}{D}
$$

\section{IMPROVEMENT SCHEME OF TRANSMISSION INTERFERENCE WITH DISTRIBUTED ANTENNAS SYSTEM}

The methods to improve transmission interference performance include reduction of message transmission frame error rate with physical layer technology (e.g. channel coding algorithm or equalization algorithm) and reduction of message transmission loss probability [6], [7]. However, these methods can not prevent the reduction of transmission interference caused by frequent handover. Distributed antennas system (DAS) can not only improve the signal-to-noise ratio in tunnels [8] but also expand cell coverage area. Therefore, we can resort to DAS technology to improve transmission interference.

The coverage scheme along the railway with distributed antennas system is shown in Fig. 3. Every radio frequency antenna is called radio access unit (RAU), and its coverage area is called radio frequency $(\mathrm{RF})$ cell. RAU only processes radio frequency signal, while base station processes base-band signal. Base station is connected with $N$ RF antennas. Radio signal use the same carrier frequency. The whole coverage area of $N$ RAUs connected to the same base station is called generalized cell. A certain range of coverage overlapping area is required among radio frequency cell and generalized cell.

\section{A. Coverage Area Analysis}

Path loss is defined as the ratio of effective power and receiving power.

$$
\overline{P L}=\frac{P_{t}}{P_{r}}
$$

where $P_{t}$ and $P_{r}$ denotes effective power and receiving power, respectively. For any distance, average large scale path loss is denoted as

$$
\overline{P L}(d)=\overline{P L}\left(d_{0}\right)\left(\frac{d}{d_{0}}\right)^{n}
$$

where $d$ denotes the distance between transmitter and receiver; $n$ denotes path loss exponent; $d_{0}$ denotes the ground proximity reference distance; $\overline{P L}\left(d_{0}\right)$ denotes the average path loss from transmitter to ground proximity reference point. The reference distance is normally $100 \mathrm{~m}$ or $1 \mathrm{~km}$ [9] under outdoor environment. Assume that $C$ is a constant, and $C=\frac{\overline{P L}\left(d_{0}\right)}{\left(d_{0}\right)^{n}}$, then the average large scale path loss of any distance is denoted as

$$
\begin{aligned}
& \overline{P L}(d)=C d^{n} \\
& d=\left(\frac{P_{t}}{C P_{r}}\right)^{1 / n}
\end{aligned}
$$

A generalized cell may replace the original cell if radio coverage area along railway employs distributed antennas. Due to the linear coverage employed by the most areas along railway, assuming that base station transmitted power is a constant, considering only large scale path loss, the coverage area of distributed antenna scheme is analyzed. Assume that a generalized cell coverage radius is denoted as $d_{D A S, N}$, and it equals to the sum of coverage radius of the $N$ RF cells which are the components of the generalized cell (coverage overlapping cells are not considered).

$$
d_{D A S, N}=\sum_{i=1}^{N} d_{i}
$$

where $d_{i}$ denotes the radius of the $i$ th RF cell of the base station, $i=1,2 \cdots N$. Under the circumstance of constant coverage level and constant cell edge communication probability requirement, assuming that transmitted power of base station is equally distributed on each antenna, then RF cell coverage radius is denoted as:

$$
\begin{gathered}
d_{i}=\left(\frac{P_{t} / N}{C P_{r}}\right)^{-1 / n}=N^{-1 / n} d \\
d_{D A S, N}=N^{1-1 / n} d
\end{gathered}
$$

Compared with single base station and single antenna coverage, distributed antennas coverage area is expanded $N^{1-1 / n}$ 
times with linear coverage, while coverage area is expanded $N^{1-2 / n}$ times with plane coverage[10].

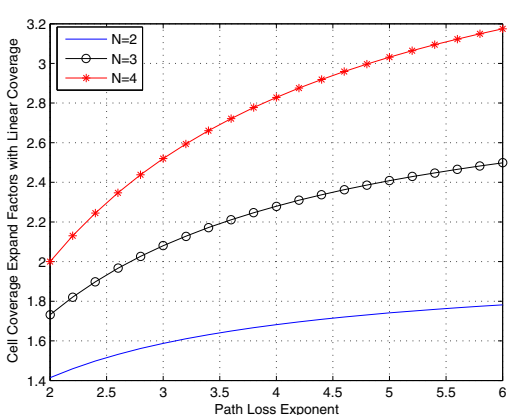

Fig. 4. Impact of path loss factor and the number of antennas on cell coverage area

Fig. 4 shows the impact of the number of antennas and path loss exponent on distributed antennas cell coverage expanding factors with linear coverage. Cell coverage area can be expanded at least 1.4 times with distributed antennas coverage. With the increasing of $N$, generalized cell coverage area increases obviously.

Collision probability with distributed antennas coverage is

$$
p_{D A S, N}=N^{1 / n-1} p
$$

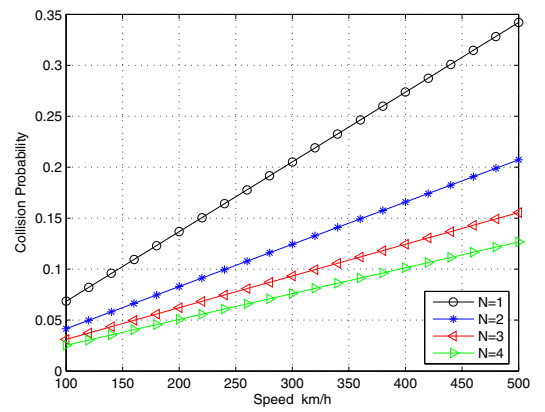

Fig. 5. Impact of the number of antennas and train speed on collision probability

TABLE I

COLLISION PROBABILITY SIMULATION PARAMETERS

\begin{tabular}{cc|cc}
\hline Parameter & Value & Parameter & Value \\
\hline$R_{I}$ & $9600 \mathrm{bit} / \mathrm{s}$ & $F E R$ & $10^{-1}$ \\
$T_{d}$ & $0.32 \mathrm{~s}$ & $T_{r}$ & $2 \mathrm{~s}$ \\
$T_{h}$ & $0.3 \mathrm{~s}$ & $T_{c}$ & $20 \mathrm{~s}$ \\
$V$ & $360 \mathrm{~km} / \mathrm{h}$ & $D$ & $2 \mathrm{~km}$ \\
\hline
\end{tabular}

Impact of train speed on collision probability under different number of antennas are compared in Fig. $5(n=3.5)$. The other parameters are shown in Tab. I. $N=1$ denotes the collision probability under single base station and single antenna system, while $N>1$ denotes the collision probability under distributed antennas system. As is shown in Fig. 5, when $N=2$, collision probability is reduced nearly by half compared with that of single antenna coverage system under the same moving speed. With the increasing number of antennas, the rate of increment is becoming small.

\section{B. Carrier Interference Ratio Analysis}

Assume that transmitted power of base station is the same between single antenna coverage system and distributed antennas system. Only co-channel interference within system is considered as interference signal. Frequency multiplexing adopts 6 frequency groups. Under the condition of linear coverage, frequency point reemerges every five cells. Available signal power received by single antenna coverage system cell edge is

$$
C_{S A}=\frac{P_{t}}{C d^{n}}
$$

Assume that radius of each cell is the same, and then cochannel interference signal power received by this cell edge is

$$
I_{S A}=\frac{P_{t}}{C(11 d)^{n}}+\frac{P_{t}}{C(13 d)^{n}}
$$

Therefore, carrier interference ratio of single antenna coverage scheme is

$$
C_{S A} / I_{S A}=(143)^{n} /\left(11^{n}+13^{n}\right)
$$

Based on the analysis in subsection A, generalized cell radius of distributed antennas scheme is $N^{1-1 / n} d$. Each RF cell radius is approximately $N^{-1 / n} d$. Therefore, carrier signal power received by generalized cell edge is

$$
C_{D A S}=\sum_{k=1}^{N} \frac{P_{t}}{C(2 N-2 k+1)^{n} d^{n}}
$$

Co-channel interference signal power is

$I_{D A S}=\frac{P_{t}}{C d^{n}}\left(\sum_{k=1}^{N} \frac{1}{(12 N-2 k+1)^{n}}+\sum_{k=1}^{N} \frac{1}{(14 N-2 k+1)^{n}}\right)$

Therefore, system carrier interference ratio is

$$
C_{D A S} / I_{D A S}=\frac{\sum_{k=1}^{N} \frac{1}{(2 N-2 k+1)^{n}}}{\sum_{k=1}^{N} \frac{1}{(12 N-2 k+1)^{n}}+\sum_{k=1}^{N} \frac{1}{(14 N-2 k+1)^{n}}}
$$

where $N$ denotes the number of radio frequency cell of generalized cell; $n$ denotes path loss exponent.

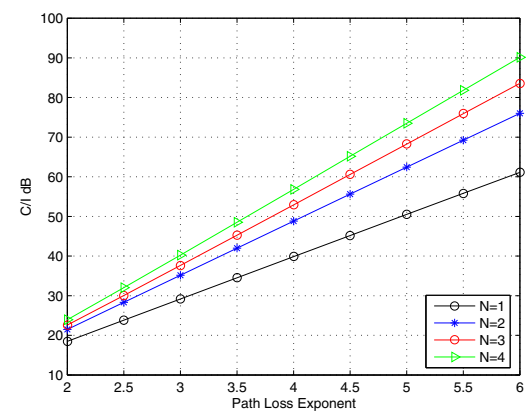

Fig. 6. Impact of distributed antennas scheme on carrier interference ratio

Under distributed antennas coverage scheme, the impact of 
the number of antennas and path loss exponent on communication system carrier interference ratio is shown in Fig. 6. Compared with single antenna coverage scheme, under the condition of path loss exponent $n=3.5$, if $N=2$, there is an 7$8 \mathrm{~dB}$ improvement for distributed antennas coverage scheme, while $N=3$ or 4 , there is only $3-4 \mathrm{~dB}$ improvement of carrier interference ratio on this basis. With the increasing of $N$, the increasing amount of improvement becomes slow.

\section{Generalized Cell Coverage Area with Restriction}

Mobile station within one generalized cell can receive several signals from different RF antennas. The information carried by these signals has the same content but different time delay when they are received. Therefore, it can be processed as a kind of multipath effect. However, when the delay of multipath effect is expanded too much, coherent bandwidth is less than modulation bandwidth, and then inter-symbol interference (ISI) will be introduced. As a result, system transmission interference performance will be degraded. Equalizer within the receiver can compensate the delay in the channel, and thus weaken the impact of intersymbol interference [9]. However, the compensation on delay with equalization technology is limited, and it is proportional to the complexity of the equalizer. For example, the maximum likelihood sequential estimation equalizer normally adopted by GSM system adopts the 16 state (Viterbi Algorithm)VA to achieve compromise between complexity and performance. This equalizer can be employed in multipath environment with a delay less than 4 code elements (approximately 15 $\mu s$ )[11]. Under such condition, there is no increase intersymbol interference in receiving signal and there is only direct path between RF antennas. The distance between the two RAUs (namely the distance between RAU 1 and RAU $\mathrm{N}$ in Fig. 3) of two ends of a generalized cell is

$$
3 \times 10^{8} \mathrm{~m} / \mathrm{s} \times 15 \mathrm{us}=4.5 \mathrm{~km}
$$

Then, the generalized cell diameter is approximately

$$
D_{D A S N}=4.5 \times \frac{N}{N-1}
$$

where $D_{D A S N}$ denotes generalized cell diameter; $\mathrm{N}$ denotes the number of radio frequency cells within generalized cell. If there are too many reflection paths because of ambient environment, generalized cell diameter should be reduced, which may reduce when the number of radio frequency cells within generalized cell increases, as is shown in Tab. II. Therefore, generalized cell coverage area is limited by

TABLE II

GENERALIZED CELL RADIUS WITH EQUALIZER CONSTRAINTS

\begin{tabular}{cccc}
\hline Number of antennas & $N=2$ & $N=3$ & $N=4$ \\
\hline Generalized cell diameter $D$ & $9 \mathrm{~km}$ & $7 \mathrm{~km}$ & $6 \mathrm{~km}$ \\
\hline
\end{tabular}

the equalizer performance within receiver. Although coverage area will increase, interference will be reduced, and carrier interference ratio will increase with the increasing of the number of antennas. Serious ISI will occur under the condition of the constant complexity of on-board receiver equalization algorithm. The high complexity of equalization algorithm will increase the processing time delay of signal, and as a result it is not suitable for train control message transmission service with higher real-time requirement. Therefore, tradeoff is necessary between the complexity and performance of equalization algorithm.

\section{CONCLUSiOn AND Future WORK}

Based on the analysis of those main elements that impact railway digital mobile communication system transmission interference, the relation between train speed and collision probability is established. In addition, we propose the scheme of distributed antennas wireless coverage along railway, and the improvement method of communication system transmission interference performance by reducing collision probability between train control message and handover. Theoretical analysis in this paper demonstrates that under the constant base station transmitting power and the path loss exponent $n=3.5$, distributed antennas coverage along railway can expand coverage area at least 1.65 times, and increasing carrier interference ratio of received signal $7-8 \mathrm{~dB}$. The proposed scheme improves transmission interference performance effectively in high speed railway.

Generalized cell coverage area is limited by receiver equalization algorithm performance. The better the performance of the equalizer, the smaller the time delay of receiving process. In the future, we will study how to coordinate distributed antennas system configuration and receiver equalization algorithm designing to achieve a better transmission interference performance of RDMCS.

\section{REFERENCES}

[1] China Tietong Qianghai Branch and Beijing Jiaotong University, "GSMR Network QoS Test Reporter of Qinghai-Tibet Line", 2008

[2] J. Xie, Z. Yang, "QoS Analysis of GSM-R for Train Control,"Railway Signalling \& Communication, vol.43, no.1,pp. 32-34, 2007.

[3] Z. Huang, X. Zhang,"Study on QoS Parameters and Measurement Approach for GSM-R Network," Railway Signalling \& Communication, vol.43, no.1, pp. 38-40, 2007.

[4] European Economic Interest Group European Rail Traffic Management System, "ETCS/GSM-R Quality of Service Operational Analysis," Reference EEIG: 04E1171, 2005.

[5] UNISIG, "ERTMS/ETCS-Class 1 GSM-R Quality of Service Class 1 Requirements", SUBSET-093 v2.2, 2005.

[6] C. Briso, C. Cortes, F. J. Arques ,J. I. Alonso, "Requirements of GSM Technology for the Control of High Speed Trains," in Proc. IEEE PIMRC'02, Lisbon, Portugal, Sep.2002, pp: 792- 793.

[7] C. Y. Farwell, R. D. Miller, and R. A. Windhausen, "Mobile- Synchronized Handover in a Wireless Communications System", United States Patent, No.5444766, 1995/8/22.

[8] C. Briso, J. M. Cruz, J. I. Alonso, "Measurements and Modeling of Distributed Antenna Systems in Railway Tunnels," IEEE Trans. veh. Technol., vol.56, no.5, pp. 2870-2879, 2007.

[9] T. Rappaport, "Wireless Communications: Principles and Practice, second editon," Englewood Cliffs, NJ: Prentice-Hall, 2001.

[10] P. Chow, A. Karim, V. Fung, and C. Dietrich,"Performance advantages of distributed antennas in indoor wireless communication systems,"in Proc. IEEE VTC'94, Stockholm, Sweden, Jun. 1994, pp. 1522-1526.

[11] F. Wang, S. Peng, "Impact Analysis on Multipath Caused by Repeater in GSM System,” Telecommunication Technology, vol.12, pp. 28-30, 2007. 